\title{
In vivo Tumor Wavelength Band Selection using Hierarchical Clustering and PCA with NIR-Hyperspectral Data
}

\author{
${ }^{1}$ Mai Kasai, ${ }^{2}$ Yuya Yasuda, ${ }^{1}$ Hiroshi Mizoguchi, ${ }^{2}$ Kohei Soga, ${ }^{3}$ Kazuhiro Kaneko, ${ }^{1}$ Hiroshi Takemura, \\ ${ }^{1}$ Department of Mechanical Engineering, Faculty of Science and Technology, Tokyo University of \\ Science, Chiba, Japan; \\ ${ }^{2}$ Department of Materials Science and Technology, Faculty of Industrial Science and Technology, \\ Tokyo University of Science, Tokyo, Japan; \\ ${ }^{3}$ Department of Gastroenterology and Endoscopy, National Cancer Center Hospital East, Japan; \\ 7515613@ed.tus.ac.jp
}

\begin{abstract}
This paper presents a new method of wavelength selection combined with principle component analysis (PCA) and a hierarchal method for hyperspectral data analysis. Hyperspectral data analysis is a combination of imaging and spectroscopic technology, and is utilized in several fields. In the medical field, if it is possible to distinguish the region of interest by selecting a feature wavelength without the intervention of manufacturers, spectral application as a surgery support system would be feasible. There are several analysis techniques to extract the features using hyperspectral data; however, many of these methods are premised on using all wavelengths. Considering application to endoscopes and other medical devices and reducing number of wavelengths, these methods are not applicable. PCA is a popular analysis for reducing the number of wavelengths, and has been adapted for extracting general and clear features of specimens. However, such extracted features from the body tend to highlight the features of protein and blood making it difficult to extract the features of cancer or other diseases. This paper proposes a two-type feature extraction method that depends on "difference" and "similarity". In this method, the PCA and hierarchal classification analysis are combined to merge the clusters based on similarity. The feasibility of the proposed method is verified by applying the method to discriminating the cancer of mouse: colonized cancer tissue after three weeks and colonized cancer tissue after one week. The results show that the proposed method can extract the two-type feature. The proposed method is had an accuracy of $75.9 \%$ in reducing the number of wavelength bands from 256 to 8 for mature cancer in mice. Although less accurate than using all wavelength bands, the eight wavelength bands are distinguished using a simplified convolutional neural network model with an accuracy of $75.9 \%$. The proposed method is able to select the feature wavelength bands for the target. As the learning model for improving the accuracy advances beyond the simple model and the new feature is extracted in the selected wavelength bands, the method is applied to a diagnosis supporting system for endoscopic surgery and more.
\end{abstract}

Keywords: PCA; Hierarchal Method; Hyperspectral Data; Band Selection; Cancer Detection. 


\section{Introduction}

Medical image processing techniques have progressed rapidly in recent years [1-2]. These techniques are supported by many innovative technologies, such as X-ray imaging, computed tomography, magnetic resonance imaging, ultrasonic tomography, and positron tomography. X-ray imaging and scintigraphy, which utilize electromagnetic rays of the invisible wavelength region, are particularly useful for clinical diagnosis. Near infrared ray (NIR), which is noninvasive to the human body, is being actively applied to medical image techniques [3-4]. NIR has the potential to be usable in medical image processing techniques in addition to $\mathrm{X}$-ray and gamma ray. More noninvasive diagnosis support system and identification technique of non-acquired lesion are possible by combining NIR with current technologies. Soga et al. proposed, as medical techniques using a spectrogram in NIR, fluorescent bioimaging techniques such as Fluorescent Probes [5] and NIR dual-functional targeting probes [6]. Such techniques generally require the use of markers to distinguish the target region as necessary for diagnosis in medical imaging. If the feature wavelength bands of the diagnosis target become available to replace the specific excitation wavelength bands of the spectrogram in probes, we can expect the diagnosis support system to be applicable without the intervention of any manufacturers or medication. With the number of wavelength bands/channels of the spectrogram reduced to extract feature wavelength bands of the target region, this has been applied to a diagnosis support system using an endoscope combined with a three-dimensional image capture system by confocal headscanning mechanism (Kondo et al.) [7-8]. Since this mechanism can use only several wavelength bands simultaneously, in this paper, we propose a new method to select the feature wavelength bands to extract the diagnosis target region from an NIR hyperspectral image. The proposed method is applicable to the development of new endoscopic imaging and diagnosis supporting systems. In order to select the specific wavelength bands in an NIR hyperspectral image, we focus the hyperspectral imaging data to acquire positional information over a two-dimensional (2-D) area and spectral data corresponding to the material of each pixel. Hyperspectral imaging data are utilized in several research fields, including soil analysis and quantitative evaluation of foods [9-13]. There are several analysis techniques to distinguish a target region using hyperspectral imaging data. These techniques include the Gabor filter, grey level co-occurrence matrix (GLCM), principle component analysis (PCA), minimum noise fraction (MNF), and wavelet transform [14-17]. In many cases, these methods assume the use of all wavelength bands of the hyperspectral image. The hyperspectral image data often have redundant information to detect the region of interest. In such cases, to consider only several wavelength bands is considerably more effective than to consider all wavelength bands. $\mathrm{Pu}$ et al. and Agarwal et al. proposed dimension reduction methods using PCA to reduce redundant information from hyperspectral imaging data [18-19]. In hyperspectral imaging data, these methods reduce the dimensions to improve the distinguishing accuracy, which is often dependent on the target. For applications to surgery support systems, the number of wavelength bands required to be reduced is dependent on the limitations of the endoscope hardware performance. Using these dimension reduction methods to improve accuracy, the accuracy is not guaranteed in the case of reducing the number of the wavelength bands of the target region. With the target located within the body where protein and blood are predominantly present, if PCA is applied to reduce dimension of the wavelength bands that are likely to extract the whole/specific feature, the results are prone to be affected by disproportionate weighing of protein and blood. 
Mai Kasai, Yuya Yasuda, Hiroshi Mizoguchi, Kohei Soga, Kazuhiro Kaneko, Hiroshi Takemura,; In vivo Tumor Wavelength Band Selection using Hierarchical Clustering and PCA with NIR-Hyperspectral Data. Journal of Biomedical Engineering and Medical Imaging, Volume 4, No 1, February (2017), pp 1-12

We propose a feature wavelength band selection method, combining a clustering method and neural network to reduce the dimensions and to improve the distinguishing accuracy. The proposed method has two steps; the dimension reduction step combines PCA [20] and a k-means clustering method [21], and the feature wavelength band selection step is based on a convolutional neural network (CNN) [2223] using hyperspectral data. The proposed feature wavelength band selection method is able to distinguish a target in the body.

\section{Proposed Method}

\subsection{Near infrared hyperspectral imaging data and calibration}

In this research, we used an NIR-hyperspectral imaging camera, Compovision (Sumitomo Electric Industries, Ltd., CV-N800HS), which can acquire spectral data of broad wavelength range and 2-D image data, to select the suitable wavelength band for the target (Fig. 1). The wavelength band resolution of the Compovision is approximately $6 \mathrm{~nm}$ from 900.00 to $2400.00 \mathrm{~nm}$, and it is a line scan camera, 320 pixel per line. Each pixel has 256 wavelength bands. To improve the analytical accuracy, the original data $V_{n}(0 \sim 216-1)$ is corrected from the dark and white data of the camera. The dark data $D$, obtained by turning off the light and covering the lens with a lens cap, is the background response of the camera. The white data $\mathrm{W}$ is obtained with a standard reflector, which is non absorbing in NIR wavelength bands, to area-correct for the wavelength sensitivity characteristic of the camera and light.

The corrected reflectance value $\mathrm{f}$ is calculated as follows:

$$
f=\frac{V_{n}-D}{W-D} \times\left(2^{16}-1\right)
$$

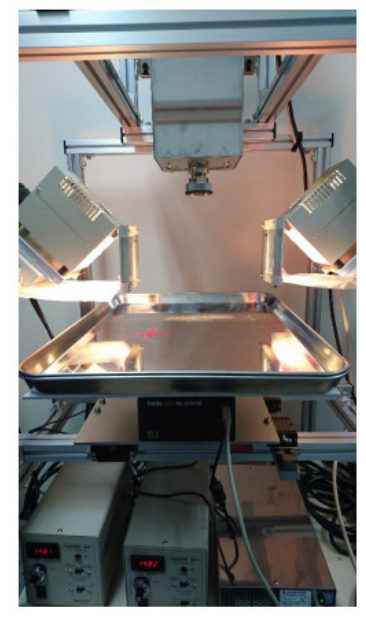

(a)

Compovision ${ }^{\circledR}$ (Sumitomo Electric Industries, Ltd., CV-N800HS)

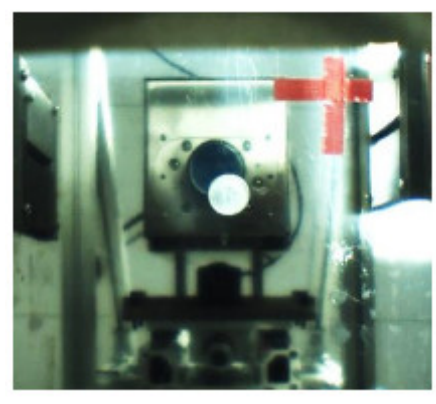

(b) Visible image

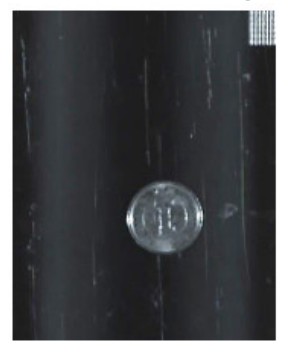

(c) NIR image

Figure. 1 image of the Compovision using this paper. (b) shows the visible image by visible camera and (c)shows the NIR image by Compovision ${ }^{\oplus}$. 


\subsection{Proposed method}

In this paper, we propose a new method of selecting the suitable feature wavelength bands, which combines dimension reduction and CNN. The flowchart of the proposed method is shown in Figure 2 . The proposed method is mainly composed of the following two steps: $(A)$ reducing dimension by PCA and k-means clustering and (B) selecting the wavelength bands by CNN.

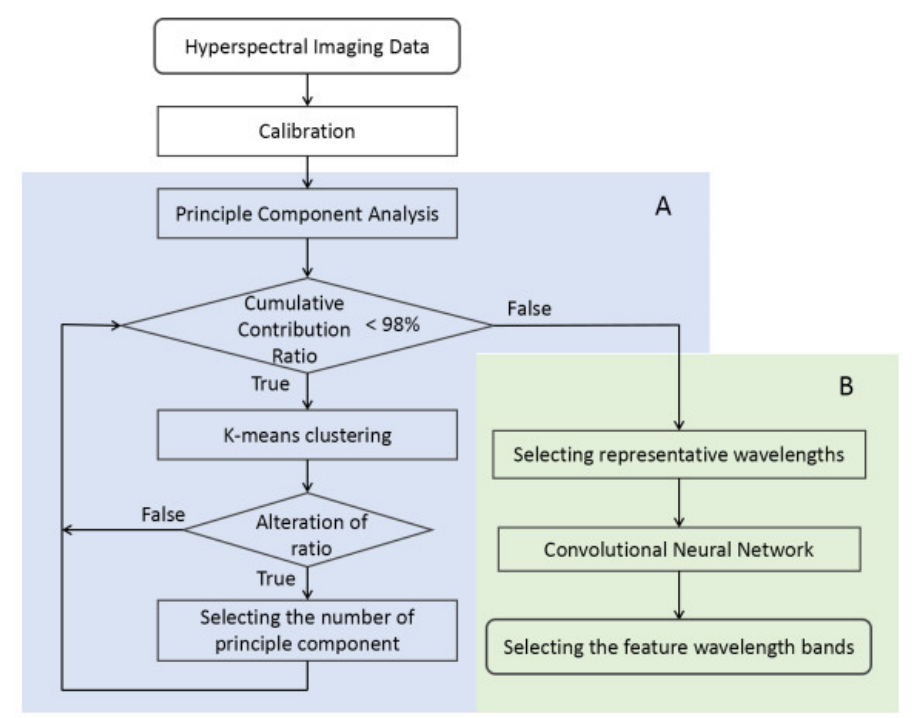

Figure 2 the flowchart shows the proposed method. The proposed method is mainly composed of the following two steps; (A) reducing dimension by PCA and k-means clustering and(B) selecting the wavelength bands by CNN.

\subsection{Reducing dimensions (A)}

The calibrated data is applied to PCA and each PCA score is calculated. PCA is a technique of multivariate analysis that reduces the dimensionality. PCA has been utilized to reduce dimension, extract features, compress data, and identify key factors. While the cumulative contribution ratio is less than $98 \%$, that process is repeated. The PCA score is then applied to the k-means clustering method and is divided over some area. K-means clustering is representative of the partitioned optimization clustering techniques that search for an optimal partition by an evaluation function. The PCA score is divided into clusters by similarity on a principle component axis. K-means clustering is not applied to each PCA score, but rather to the PCA score derived from the 1st principle component to the $n$th principle component. This process prevents duplication of the extracted features and is more effectively able to reduce the wavelength bands. If the cluster region is changing, compared to the resulting visualized PCA score by k-means clustering, the number of principle component axis (NPCA) is selected. If the difference between the clustering result for the image of the $i$ th and that of $(i+1)$ th principal components satisfies the following conditions: a change of cluster implies that a different is valued by number of pixels and is more $20 \%$ than result of pre-image, the $(i+1)$ th principle component that contained feature information is selected to divide the image region. 
Mai Kasai, Yuya Yasuda, Hiroshi Mizoguchi, Kohei Soga, Kazuhiro Kaneko, Hiroshi Takemura,; In vivo Tumor Wavelength Band Selection using Hierarchical Clustering and PCA with NIR-Hyperspectral Data. Journal of Biomedical Engineering and Medical Imaging, Volume 4, No 1, February (2017), pp 1-12

\subsection{Selecting wavelength bands}

\subsubsection{Selecting representative wavelength bands}

The representative wavelength band is selected by the selecting NPCA at process A in the dimension reduction. The representative wavelength band is comprised of a maximum of three selected wavelength bands as significant peak values of NPCA, according to a loading factor value as the following equation.

$$
\begin{aligned}
F_{n}> & F_{\max } \times 0.9 \\
& \text { If }(\operatorname{len}(n)>3) \text { Select top three } F_{n},
\end{aligned}
$$

where $F_{n}$ is a loading factor value at a wavelength band, $F_{\max }$ is the maximum value of the loading factor value and len () is an element-counting function. In PCA, the loading factor indicates the correlation between the principle component and the identified the factor that affects each principle component. The loading factor values of the selected principle component are compared and the peak wavelength band is extracted as feature values. If the loading factor value does not show a significant peak, the loading factor is regarded as not having strongly concerned the principle component and no wavelength band is extracted from this axis. The representative wavelength band is the group of selected wavelengths for each NPCA. If the region of the result image does not change as a whole, the representative wavelength band is the group of wavelengths that satisfies the maximum value of the loading factor value for every principle component.

\subsubsection{Selecting the wavelength using CNN}

To select a more suitable wavelength band from the representative wavelength bands for distinguishing the target, the proposed method uses mini batch learning by CNN. CNN is the technique of deep learning based on perception in the human visual cortex. To apply the same method if the number of wavelengths is changed, spectral data of 256 wavelength bands per pixel is converted into a $16 \times 16$ image and CNN is applied to this image (Figure. 3). When wavelength bands are reduced, the data gap is interpolated by Lagrange interpolation. The value calculated by equation (3) and the value between edge points $(0,0),(255,0)$ are fixed.

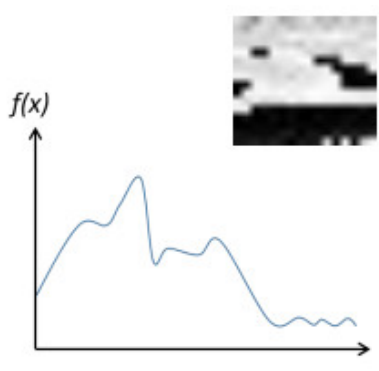

(a) using all wavelehgth bands

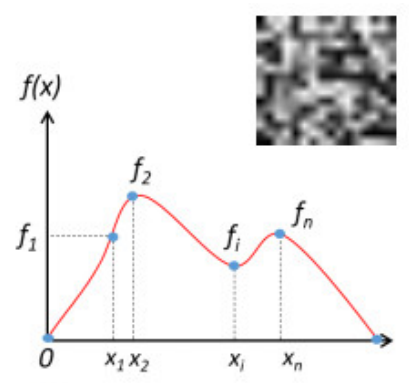

(b) using selected wavelength bands

Figure. 3 spectral data of 256 wavelength bands per pixel is converted into $16 *^{*} 16$ image and this image is applied is used for CNN (a). when wavelength bands are reduced, the data gap interpolates by Lagrange interpolation interpolates the data gap (b) 


$$
F(x)=\sum_{i=1}^{n+1} y_{i} \frac{f_{i}(x)}{f_{i}\left(x_{i}\right)}
$$

where $f_{i}(x)=\prod_{k \neq 1}\left(x-x_{k}\right)$ and if the number of wavelength bands used is $m, n=m+1$. Considering using a method such as machine learning $(\mathrm{ML})$ to improve accuracy for application after selecting the feature wavelength bands, a simplified model of CNN is used in this wavelength band selection. The model consists of two repeated two cycles of convolution and pooling layers and is termed multi-layer perception. This model uses two-class classification to distinguish whether the target is cancer or not. The batch size is 100 , the epoch number is 40 , and the model is shown in Figure 4 . The accuracy by CNN is compared with many patterns that change number of wavelength bands or combination of wavelength bands in the representative wavelength selection. The wavelength pattern with the highest accuracy is regarded as the feature wavelength band. This method is able to select a more suitable wavelength band for distinguishing the target region.

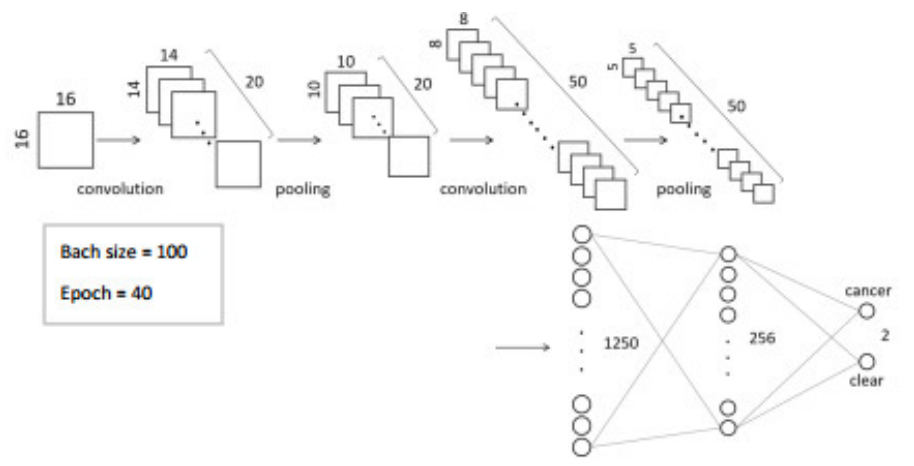

Fig. 5 The learning model of CNN using the proposed method. The model is repeated two times of convolution and pooling layer and sited multilayer perceptron. This model is two class

classification to distinguish whether cancer or not. Bach size is 100 , epoch is $\mathbf{4 0}$

\section{Experiment}

In order to assess the proposed method, we experimented to distinguish the cancer area of a mouse using the feature wavelength bands from the method. This study received the approval of the ethics committee of Tokyo University of Science. Targets are divided into Dataset 1 and Dataset 2 (Fig. 5). Dataset 1 consists of data from five mouse specimens of colonized cancer tissue and Dataset 2 consists
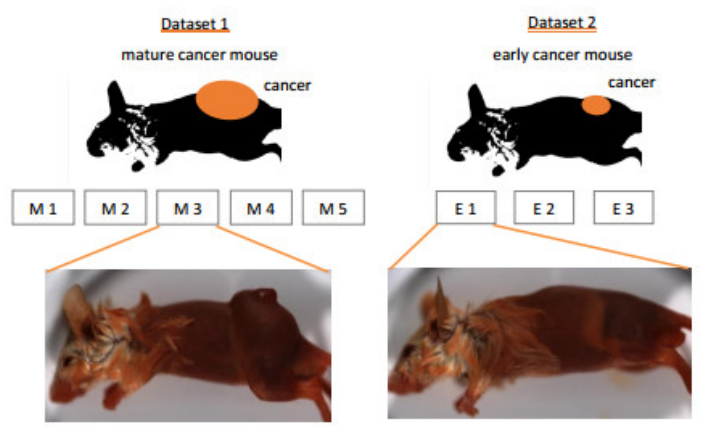

Fig. 6 Targets in this experiment are divided with Dataset 1 and Dataset 2. Dataset 1 is 5 mouse data of colonized cancer tissue and Dataset $\mathbf{2}$ is $\mathbf{3}$ mouse data of colonized cancer tissue after $\mathbf{1}$ week. 
Mai Kasai, Yuya Yasuda, Hiroshi Mizoguchi, Kohei Soga, Kazuhiro Kaneko, Hiroshi Takemura,; In vivo Tumor Wavelength Band Selection using Hierarchical Clustering and PCA with NIR-Hyperspectral Data. Journal of Biomedical Engineering and Medical Imaging, Volume 4, No 1, February (2017), pp 1-12

of data from three mouse specimens of colonized cancer tissue after one week. In these experiments, we acquired hyperspectral image data of dimension 320 (pixels per line) $\times 234$ (wavelength bands per pixel) $\times 720$ (number of lines).

\subsection{Distinguishing mature cancer of mouse (A)}

The data of each mouse in Dataset 1 are applied to the proposed method. After selecting the feature wavelength bands of each mouse, the learning model is created by the wavelength band. The accuracy of the created model is calculated by test data using the other mouse data in Dataset 1 . The average accuracy at the other four mouse data defines the accuracy of the feature wavelength band. Comparing the acquired accuracy data, the feature wavelength band that has the highest accuracy is the feature wavelength band for this experiment.

\subsection{Distinguishing early cancer of mouse (B)}

The feature wavelength bands acquired by experiment $A$ are applied to all data of Dataset 1 and the learning model is created. The mouse data of Dataset 2 is distinguished of early cancer area using the model by Dataset 1 .

\section{Experimental Results}

\subsection{Distinguishing mature cancer of mouse}

Figure 6 and table 2 show the results of distinguishing the cancer area of each mouse of Dataset 1 by the proposed method. The distinguished cancer pixels are painted in color and the clear pixels represent the region not affected by cancer. M1-M5 are the mouse numbers of Dataset 1 . The feature wavelength band of each data by the proposed method is selected. Mouse number identifies the mouse used to select the feature wavelength band and to create the learning model. In this experiment, accuracy is used as a metric for the sensitivity of detecting cancer. The accuracy is calculated by as the ratio of true results, as distinguished by CNN whether each pixel is cancer, as shown in equation (4)

$$
\text { accuracy }=\frac{\mathrm{TP}+\mathrm{TN}}{T P+T N+F P+F N}
$$

Table 1 the table of confusion matrix

\begin{tabular}{|l|c|c|c|}
\hline \multicolumn{2}{|c|}{} & \multicolumn{2}{c|}{ Answer } \\
\cline { 3 - 4 } \multicolumn{2}{|c|}{} & Cancer & Clear \\
\hline \multirow{2}{*}{ Recognize } & Cancer & True Positive (TP) & False Positive(FP) \\
\cline { 2 - 4 } & Clear & False Negative(FN) & True negative(TN) \\
\hline
\end{tabular}

The average accuracy of the other four data that are not used to select the feature wavelength band and create learning model is shown. Table 2 shows that the accuracy of M4 is highest in this experiment. The eight wavelength bands acquired by the proposed method are the feature wavelength band in this experiment. 
Table 2 result of accuracy and number of wavelength bands that acquired by proposed method

\begin{tabular}{|c|c|c|c|c|c|}
\hline & M1 & M2 & M3 & M4 & M5 \\
\hline $\begin{array}{c}\text { The number of } \\
\text { Wavelength bands [-] }\end{array}$ & 7 & 8 & 9 & 8 & 10 \\
\hline Accuracy [-] & 0.300 & 0.298 & 0.456 & 0.759 & 0.478 \\
\hline
\end{tabular}

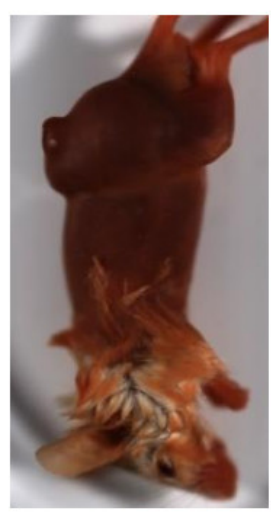

(a)

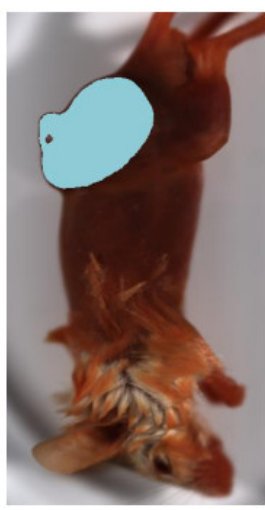

(b)

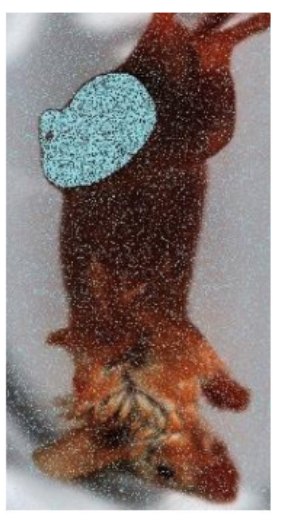

(c)

cancer area of answer

cancer area of distinguished by eight wavelength bands.

Fig. 7 result image of distinguished mature cancer area of mouse by the proposed method. (b) is answer area given the learning model as label, and (c) is result of recognized cancer area using eight wavelength bands by proposed method.

\subsection{Distinguishing early cancer of mouse}

Figure 7 and table 3 show the result of distinguishing the early cancer area of Dataset 2 using the feature wavelength bands acquired in experiment $A$. The result is evaluated by accuracy (Equation 4). ALLSP is the result of the early cancer area of Dataset 2 using the learning model created by all the data of Dataset 1, and all 256 wavelength bands.

DIVSP is the result using the 8 feature wavelength bands by the proposed method. E1-E3 indicate the mouse number of Dataset 2.

Table 3 result of accuracy using all wavelength bands "ALLSP" and selected wavelength bands by proposed method "DIVSP"

\begin{tabular}{|c|c|c|c|}
\hline Accuracy [-] & E1 & E2 & E3 \\
\hline DIVSP & 0.156 & 0.179 & 0.272 \\
\hline ALLSP & 0.966 & 0.968 & 0.974 \\
\hline
\end{tabular}


Mai Kasai, Yuya Yasuda, Hiroshi Mizoguchi, Kohei Soga, Kazuhiro Kaneko, Hiroshi Takemura,; In vivo Tumor Wavelength Band Selection using Hierarchical Clustering and PCA with NIR-Hyperspectral Data. Journal of Biomedical Engineering and Medical Imaging, Volume 4, No 1, February (2017), pp 1-12

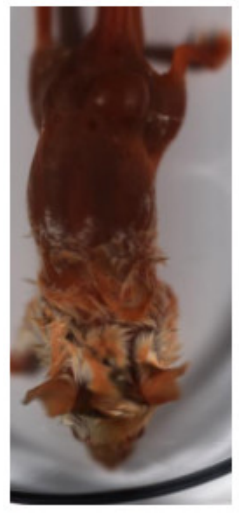

(a)

NIR image

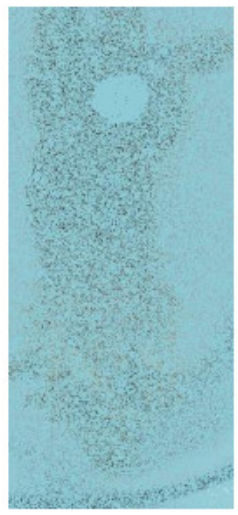

(b)

cancer area of distinguished

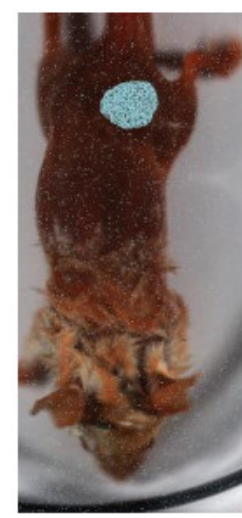

(c)

cancer area of distinguished by eight wavelength bands. by 256 wavelength bands.

Fig. 8 result image of distinguished early cancer area of mouse by the proposed method.

(b) is result eight wavelength bands by proposed method, and (c) is result of recognized cancer area using all wavelength bands (256 wavelength bands).

\section{Discussion}

Table 2 shows that if the feature wavelength band is selected by the proposed method, the accuracy is approximately $46 \%$ in the mature cancer mouse. As the proposed method selects five patterns of feature wavelength band, the eight wavelength bands of M4 most clearly distinguished the cancer area and the accuracy is $75.9 \%$. The result of using all wavelength bands maintained an average of 97\%. Although less accurate than using all wavelength bands, the eight wavelength bands are distinguished using a simplified CNN model and achieved an accuracy of 75.9\%. This result suggests that if the learning model is created for extracting the features of the selected eight wavelength bands, the accuracy could be improved. The data used in this experiment were acquired at different angles and in different individuals. Since the accuracy is different, the feature wavelength band selected by the proposed method had 3-4 wavelength bands in common and contained wavelength bands different from one another. The proposed method extracted the feature in the same species and in the applied individual. The lower accuracy of the clear area is attributed in part to the lower accuracy of CNN. Figure 7 shows that the clear area is not truly distinguished compared with the cancer area. As the employed wavelength band was selected to distinguish the cancer in mice, the clear area information was lost.

Table 3 shows that if the selected eight wavelength bands in experiment A are applied, distinguishing the early cancer area from the mature cancer area has an accuracy of $20.2 \%$. Comparing the accuracy from using all wavelength bands to that using eight wavelength bands, the accuracy is shown to be drastically lowered. However, considering Fig. 8, the accuracy of distinguished cancer area is increased using the selected wavelength bands. Table 4 shows the results of recall and precision. 
Table 4 result of recall and precision as each mouse data of Dataset 2

\begin{tabular}{|c|c|c|c|c|c|c|}
\hline & \multicolumn{3}{|c|}{ DIVSP } & \multicolumn{3}{c|}{ ALLSP } \\
\hline & E1 & E2 & E3 & E1 & E2 & E3 \\
\hline Recall & 0.994 & 0.995 & 0.996 & 0.805 & 0.552 & 0.458 \\
\hline Precision & 0.022 & 0.013 & 0.006 & 0.355 & 0.212 & 0.091 \\
\hline
\end{tabular}

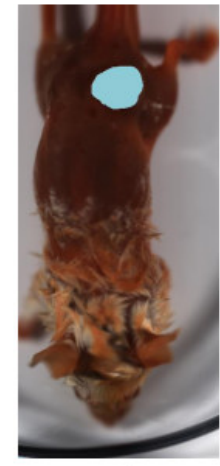

(a)

Cancer area of answer

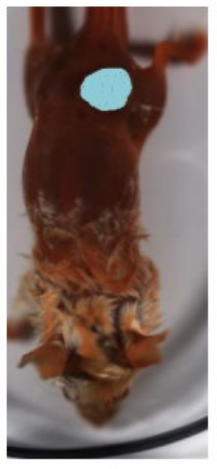

(b)

cancer area of distinguished

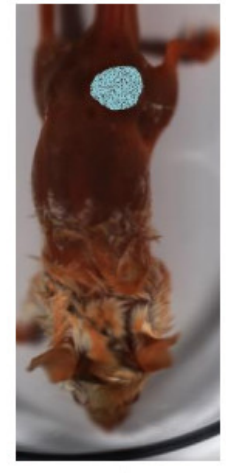

(c)

cancer area of distinguished by eight wavelength bands. by 256 wavelength bands

Fig. 9 result image of distinguished early cancer area of mouse by the proposed method. (a) is answer area given the learning model as label, (b) is result of recognized cancer area using eight wavelength bands by proposed method and (c) is result of recognized cancer area using all wavelength bands.

Recall and precision are calculated by equation 5

$$
\begin{gathered}
\text { recall }=\frac{\mathrm{TP}}{T P+F N} \\
\text { precision }=\frac{\mathrm{TP}}{T P+F P}
\end{gathered}
$$

Table 4 shows that the accuracy of distinguished early cancer area increases drastically by using the selected eight wavelength bands by the proposed method as compared to using all wavelength bands. The ratio of false positive, i.e., identifying clear area as cancer, is higher. This is true in the cases of using the all wavelength bands and the selected wavelength bands. This suggests that early cancer and clear area had more similar features than mature cancer and clear area. In these, as the distinguishing accuracy is increased in cancer itself, the selected wavelength bands can remove redundant information to distinguish between cancer and clear area. The proposed method positively extracts the wavelength bands to distinguish cancer. These results suggest that interpolation of distinguished clear area may possibly improve the algorithm that removes the scattered pixels of distinguished cancer and to improve the learning model. The two-step method that extracts the features after the wavelength band selection may possibly improve the supporting system. 
Mai Kasai, Yuya Yasuda, Hiroshi Mizoguchi, Kohei Soga, Kazuhiro Kaneko, Hiroshi Takemura,; In vivo Tumor Wavelength Band Selection using Hierarchical Clustering and PCA with NIR-Hyperspectral Data. Journal of Biomedical Engineering and Medical Imaging, Volume 4, No 1, February (2017), pp 1-12

\section{Conclusion}

We proposed a new method combining dimension reduction and CNN to select the feature wavelength bands for the target region. The proposed method had an accuracy of $75.9 \%$ to reduce the number of wavelength bands to 8 from 256 for mature cancer in mice. Although less accurate than the procedure using all wavelength bands, the procedure using eight wavelength bands can be distinguished using a simplified CNN model and achieved $75.9 \%$ accuracy. Improving the learning model and extracting the features in the selected wavelength bands may possibly further increase the accuracy. As distinguishing the early cancer using the mature cancer data, the whole accuracy is decreased considerably. The accuracy of distinguishing early cancer area is increased with respect to using all wavelength bands and is $99 \%$. This indicates that as early cancer has more similar features to clear cell than mature cancer, the accuracy of the clear area is decreased. The proposed method positively extracted the wavelength bands to distinguish the target as cancer. The distinction among different stages cancer and visible difference in individuals suggests that it is possible to increase the accuracy. If the method is improved to a two-stage analysis that extracts the features after selecting the wavelength bands, the accuracy of distinction is enhanced. The proposed method is able to select the feature wavelength bands for the target. As the learning model for improving the accuracy is implemented beyond the simple model and new features are extracted in the selected wavelength bands, the method may be applied to a diagnosis supporting system for endoscopic surgery and more.

\section{REFERENCES}

[1] Matthias Nahrendorf, Hanwen Zhang, Sheena Hembrador, Peter Panizzi, David E. Sosnovik, Elena Aikawa, Peter Libby, Filip K. Swirski, Ralph Weissleder, Nanoparticle PET-CT Imaging of Macrophages in Inflammatory Atherosclerosis, Circulation, 2008, Volume 117, Issue 3.

[2] Hsiang-Yang, MaZhuhuang Zhou, Shuicai Wu, Yung-Liang Wan, Po-Hsiang Tsui, A Computer-Aided Diagnosis Scheme For Detection Of Fatty Liver In Vivo Based On Ultrasound Kurtosis Imaging, Journal of Medical Systems, Vol. 40, No. 33, 2016, pp. 6-9.

[3] Hayakawa, Y., Yamashita, H., Otuburai, T., Mitaseta, Y., Sagawa, M., Kondo, A., Tsuji, Y., Honda, A., Nearinfrared radiation imaging for the detection of alien substances under the skin, 医用画像情報学会雑誌, Vol.27, No.3， 2010, pp.50-54.

[4] Mitsunaga , M., Ogawa, M., Kosaka, N., Rosenblum, L., T., Choyke, P., L., Kobayashi, H., Cancer cell-selective in vivo near infrared photo immunotherapy targeting specific membrane molecules, Nature Medicine, 2011, pp. 1685-1691.

[5] Kohei S., Kimikazu T., Kosuke T., Tomoyoshi Y., Hiroshi H., Hidehiro K., NIR Bioimaging development of Liposome-Encapsulated, Rare-Earth Doped Y203 Nanoparticles as Fluorescent Probes, EUROPEAN JOURNAL OF INORGANIC CHEMISTRY, Vol. 18, 2010, pp. 2673-2677.

[6] Zheng X., Xing D., Zhou F., Wu B., Chen WR., Indocyanine green-containing nanostructure as near infrared dual-functional targeting probes for optical imaging and photothermal therapy, Mol Pharm, Vol. 8, 2010, pp. 447-456.

[7] Komoriya, K., Takemura, H., Mizoguchi, H., Soga, K., Hyodo, H., Kishimoto, H., Kaneko, K., NIR-fluorescent Imaging by Head-Scanning Mechanism for Near-infrared Endoscope, 生体医工学,Vol.51,No.2, pp.135 $141,2013$. 
[8] Yoshiniru, K., Komoriya, K., Takemura, H., Mizoguchi, H., Soga, K., Kishimoto, H., Kaneko, K., Development of 3-D Image Capture System by Confocal Head-Scanning Mechanism for NIR endoscope, LIFE2014 講演論 文集, 2014, P-01.

[9] 安藤正哉, 荒川正幹, 船津公人, 可視及び近赤外スペクトルを用いた土壌成分值予測モデルの構築, Journal of Computer Aided Chemistry, Vol. 10, 2009, pp. 53-62

[10] Schultz R. A., Nielsen T., Zavaleta J. R., Ruch R., Wyatt R., Gar ner H. R., Hyperspectral Imaging: A Novel Approach for Microscopic Analysis, Cytometry, Vol. 43, Issue 4, 2001, pp. 239-247.

[11] 山下洋輔, 荒川正幹, 船津公人, 近赤外スペクトルを用いた果物の内部品質解析，Journal of Computer Aided Chemistry, Vol. 12, 2011, pp. 37-46

[12] Shuangting Wang, Chunyang Wang, Research on dimension reduction method for hyperspectral remote sensing image based on global mixture coordination factor analysis, The International Archives of the Photogrammetry, Remote Sensing and Spatial Information Sciences, Volume XL-7/W4, 2015

[13] Vasques, G., M., S. Grunwald, J. O. Sickman, Comparison of multivariate methods for inferential modeling of soil carbon using visible/near-infrared spectra, Geoderma, 146, 2008, pp.14-25.

[14] Amgren M., Ha nsen PW., Eriksen B., Larsen J., Larsen R., Analysis of Pregerminated Barley using Hyperspectral Image Analysis, Journal of Agricultural and Food Chemistry, Vol. 59, 2011, pp. 11385-11394.

[15] Ataushi Y., Takumi K., Kenji W., Kenji I., Tadaaki H., Nobuyuki O., Cancer Detection from Biopsy Images using Probabilistic and Discriminative Features, 2011 18th IEEE International Conference on Image Processing(ICIP), pp. 1609-1612.

[16] Serranti S., Cesare D., Marini F., Bonifazi G., Classification of oat and groat kernels using nr hyperspectral imaging, Talanta, Vol. 103, 2013, pp. 276-284.

[17] Naganathan G. K., Grimes L. M., Subbiah J., Calkins C. R., Samal A., Meyer G. E., Visible/Near-infrared Hyperspectral Imaging for Beef Tenderness Prediction, Computers and Electronics in Agriculture, Vol. 64, 2008, pp. 225-233.

[18] Pu, R., Kelly, M., Anderson, G.L., Gong, P., 2008.Using CASI Hyperspectral Imagery to Detect Mortality and Vegetation Stress Associated with a New Hardwood Forest Disease, Journal of Photogrammetric Engineering and Remote Sensing,74(1), pp.65-75.

[19] Agarwal, A., El-Ghazawi, T., El-Askary, H., Le-Moigne, J., Efficient hierachical-PCA dimension reduction for hyperspectral imagery, IEEE International Symposium on Signal Processing and Information Technology, 2007, pp. 353-356.

[20] Pearson, K., On lines and planes of closest fit to systems of point in space, Philosophical Magazine, Vol. 2, 1901, pp.559-572.

[21] MacQueen, J., Some methods for classification and analysis of multivariate observations, Proceedings pf the Fifth Berkeley Symposium on Mathematical Statistics and Probability, Vol. 1: Statistics, 1967, pp.281-297.

[22] Yann, L., Leon, B., Yushua, B., Patrick, H., Gradient-based Learning Applied to Document Recognition, Proceedings of the IEEE, 1998, pp. 2278-2324.

[23] D. Hubel and T. Wiesel. Receptive fields of single neurones in the cat's striate cortex. The Journal of physiology, No. 148, 1959, pp. 574-591. 